\title{
La relación del Capital Humano en la Empleabilidad de los egresados en Administración de la UNMSM
}

Luis Cuba Rosales ${ }^{1}$

\begin{abstract}
RESUMEN
El presente trabajo de investigación de tipo correlacional planteó resolver el problema entre el capital humano, entendido como la formación educativa, y la empleabilidad de los egresados de la carrera profesional de administración. En esa línea, por capital humano se entiende la formación educativa, expresada en número de grados y capacitaciones obtenidas, así como la experiencia laboral, expresada en años. Mientras que por empleabilidad se entiende, el salario, el tiempo que se demora en conseguir un empleo, un aumento salarial o un ascenso.
\end{abstract}

Asimismo para el trabajo de campo, se emplearon encuestas que permitieron recabar información sobre las capacitaciones recibidas y el nivel de ingresos salariales, entre otros, que los egresados tienen. Además el diseño observacional y el análisis estadístico muestra evidencia de la relación existente entre los indicadores de ambas variables. A partir de los resultados de impacto la Universidad puede contribuir ampliamente a la empleabilidad de sus egresados mediante el ofrecimiento de programas que requieren ellos de forma apremiante.

Palabras-claves: Capital humano; empleabilidad; administración.

INFLUENCE OF HUMAN CAPITAL ON EMPLOYABILITY OF MANAGEMENT GRADUATES FROM SAN MARCOS UNIVERSITY

\section{ABSTRACT}

The present correlational type research project aimed to solve the problem between human capital, understood as educational training, and the employability of graduates of the professional career of administration. In this line, human capital is understood as educational training, expressed in the number of degrees and training obtained, as well as work experience, expressed in years. While for employability is understood, the salary, the time it takes to get a job, a salary increase or a promotion.

Also for the field work, surveys were used to gather information about the training received and the level of salary income, among others, that the graduates have. Thus, the observational design and the statistical analysis show evidence of the relationship between the indicators of both variables. Based on the results of impact, the University can make a large contribution to the employability of its graduates by offering programs that they urgently need.

Keywords: Human capital; employability; administration.

\section{INTRODUCCIÓN}

La investigación abordó la relación entre el capital humano y la empleabilidad, se ha enmarcado dentro de la gestión del talento humano.

Por tanto, podemos entender que la calidad de vida de una persona depende mucho de su empleo actual, el mismo que le provee de los recursos económicos y la satisfacción profesional por su desempeño. Sin embargo, ¿cuál es la influencia que ejercen los estudios y la experiencia laboral para tener un mejor empleo? ¿Qué tanto permite el capital humano tener una adecuada gestión del empleo, o también llamado empleabilidad? Ahondar, en esta respuesta es el motivo principal del presente artículo. La población de estudio estuvo integrada por los egresados de la Facultad de Ciencias Administrativas de la Universidad Nacional Mayor de San Marcos (UNMSM), de la carrera profesional de Administración por ser una de las carreras con mayor demanda y a la UNMSM por ser una de las universidades referentes del país.

\section{Antecedentes:}

Esta investigación cuenta con antecedentes según Tito, Pereda y Vilcabana $(2008$, p. 59$)$ señalaron que "el $53 \%$ de los encuestados manifestaron que los conocimientos impartidos en la UNMSM sirvieron poco o medianamente para su desempeño laboral óptimo."

\section{En su investigación Romero y Chavarría (2009) precisaron que:}

Al egresar de la universidad, la principal preocupación del estudiante es lograr incorporarse en el mercado laboral, luego de haber logrado este cometido, el sanmarquino continúa diversos estudios para completar su formación. Solo el $6.7 \%$ de los egresados encuestados manifestó que no se encuentra trabajando actualmente. Las características del primer trabajo son a menudo muy difíciles, generalmente, el ingreso se da como practicante, en muchos casos, las labo-

1 Email: 1cubaro@sunat.gob.pe Institución: Superintendencia Nacional de Aduanas y Administración Tributaria Biografía del autor: Magister en Gestión de Organizaciones por la Universidad Bourdeaux de Francia y Licenciado en Administración de Negocios Internacionales por la Universidad Nacional Mayor de San Marcos (UNMSM). Con experiencia en la gestión pública, trabajó en la Secretaría General de la UNMSM y actualmente labora en la Superintendencia Nacional de Aduanas y Administración Tributaria (SUNAT). 
res encomendadas no guardan relación con los conocimientos y/o habilidades adquiridas, son mal remuneradas, etc. Es por ello que el grado de satisfacción con el primer empleo es relativamente bajo. Sin embargo, los resultados de la encuesta muestran una gran diferencia entre el primer empleo y el trabajo actual; pues en el empleo actual, la mayoría es contratada, desempeña el cargo de asistente (aunque un reducido porcentaje tiene ya alguna jefatura a su cargo) y el salario supera los dos mil soles en promedio. Todo ello influye en que la mayoría se encuentra bastante satisfecha con el actual empleo. (p.30)

\section{Objetivo:}

Explicar la empleabilidad del egresado de la facultad de Administración de la Universidad Nacional Mayor de San Marcos a partir de su capital humano.

- Demostrar la relación entre las capacitaciones recibidas y el rango de ingresos percibido por el egresado de la facultad de Administración de la Universidad Nacional Mayor de San Marcos.

- Demostrar la relación entre la experiencia laboral del egresado de la facultad de Administración de la Universidad Nacional Mayor de San Marcos.

\section{Justificación teórica}

El presente artículo busca presentar las premisas teóricas acerca del capital humano, y explicar a través de ésta su influencia en la empleabilidad actual para el egresado de una carrera profesional de una universidad pública. El propósito teórico es dar a conocer la importancia categórica del capital humano para el ejercicio de una profesión como de su éxito laboral, su alcance se encuentra limitado al ámbito profesional de las carreras de ciencias sociales circunscrito a la realidad nacional de un país y a sus índices de empleabilidad y estabilidad laboral, esto contribuirá a profundizar a nivel académico las variables que puedan influenciar de igual forma en su empleabilidad.

\section{Justificación práctica}

Cada persona que egresa de una universidad pública en el Perú debe contar con una formación competente que le permita acceder a oportunidades laborales en igual de condiciones que un egresado de una entidad particular. El propósito práctico es dar a conocer la realidad actual en cuanto al capital humano del egresado de una universidad pública y determinar la influencia que tiene ésta con su empleabilidad a manera de conocer su éxito laboral, esto contribuirá a la comunidad de egresados y a su vez a la toma de decisiones por parte de las órganos y entidades correspondientes.

\section{Alcance}

El presente trabajo tiene como alcance a los egresados de la facultad de Administración de la Universidad Nacional Mayor de San Marcos. Por tanto, es posible que con los resultados, conclusiones y recomendaciones se pueda inferir la realidad de las facultades de Ciencias Administrativas de la ciudad de Lima Metropolitana, con énfasis especial en las facultades nacionales. De lo cual se deduce que para aplicar estos resultados y conclusiones a las facultades de enseñar Administración del interior del país será necesario aplicar estudios complementarios que consideren la realidad en la cual se encuentran. La investigación es transversal. Esta investigación se dio en un macro entorno con relativa estabilidad.

\section{Limitaciones de la investigación}

La presente investigación, como toda pesquisa tiene las siguientes limitaciones:

- Similarmente, realizar repetidas preguntas, genera que los egresados sean reacios a contestar dos veces el mismo cuestionario, especialmente porqué se preguntan sobre temas muy delicados, como los ingresos salariales, jerarquía en el trabajo y datos personales.

- Otra limitación, es que la recolección de datos se dio por medios virtuales, y no todos acceden con frecuencia a las redes sociales. El acceso a la muestra se dio por medios virtuales, debido a la facilidad en tiempo y costo para levantar la información. Dado que ubicar a los miembros de la muestra de forma personal es muy difícil.

\section{Marco teórico}

Uno de los primeros en proponer el término capital humano fue el premio nobel de Economía, Arthur L. Theodore Schultz en 1979, para quién el capital humano es "una forma de capital porque es una fuente futura de ingresos y o de futuras satisfacciones o ambas, y es humano porque es parte integral del hombre".

Asimismo, se debe enfatizar que el "capital humano, se está refiriendo a variables que directamente se relacionan con las personas y las condiciones 
para su mejor desenvolvimiento presente y futuro" (Riveros y Baéz, 2014, p.11)

Para el también premio nobel de Economía 1992, Becker, G. (2002) el capital humano está relacionado con los conocimientos, las ideas, las habilidades, y obviamente la salud de los individuos.

Es decir, "recoge los conocimientos, aptitudes, motivación, formación, etc., de los trabajadores." (López, Nevado y Baños, 2008, p.54)

En este contexto, "las instituciones universitarias juegan un rol clave para la sociedad del conocimiento, forman el capital humano requerido por esta sociedad" (Ananias 2014 p. 273). Por ello, "La educación superior es el determinante estructural de la creación y difusión del conocimiento, de la formación y consolidación del capital humano avanzado" (Rodríguez y Palma, 2009, p.10)

Asimismo, "La inversión continua en el capital humano, es un elemento clave para promover el desarrollo económico" (Paravic, 2010, p.11) dado que "La inversión en capital humano adicional, haría más productivo a un trabajador en el futuro y, por tanto, le permitiría tener un mayor potencial de ingresos." (Améstica, Llinas y Sánchez, 2014, p.24)

Por tanto, se señala a manera de síntesis que el capital humano principalmente hace referencia principalmente al conocimiento, la experiencia y las habilidades personales.

Además del capital humano, en esta investigación se aborda el concepto de empleabilidad que "surgió a principios del siglo XX" (Mallado et al, 2016, p. 55)

En tanto, por empleabilidad se entiende como "la competencia que posee una persona para diseñar su carrera profesional, acceder al mercado de trabajo y gestionar su propio desarrollo laboral con acierto y satisfacción." (Martínez González J., 2011, p. 1) Así, la empleabilidad se entiende como aquello que "faculta al individuo para aprovechar plenamente las oportunidades del cambiante mercado laboral." (Conferencia de Ministros europeos responsables de educación superior, 2009, p. 3)

Para Ramírez (2007), empleabilidad es:

La posibilidad de las personas para encontrar un empleo que retribuya sus capacidades laborales, por medio de diferentes influencias y fuentes, tales como: Las actividades de aprendizaje organizado por medio de la educación formal e informal, por medio del entrenamiento desarrollado en los diferentes puestos de trabajo de las organizaciones, de acuerdo con cada individuo y al contexto de uso. (p. 4)

Aunque el "propio sujeto es el primer responsable de su propia empleabilidad, qué duda cabe de que la familia, el sistema educativo y las empresas, entre otras instituciones y agentes, también son responsables del desarrollo de la competencia para la carrera profesional." (Martínez González, 2012, p. 1)

Geeregat, Cifuentes y Villarroel (2016) desarrollaron la idea anterior considerando que:

La "empleabilidad reúne una serie de factores dinámicos que han sido clasificados en externos al sujeto e internos a él. Algunos externos son el prestigio de la universidad, la influencia del establecimiento educacional de origen y el salario. Entre los factores internos, se describe la influencia de los resultados en pruebas estandarizadas, la adaptación al contexto y la percepción de autoeficacia, entre otras. " (p.1)

Teniendo como referencia las definiciones relacionadas a la empleabilidad para fines de la investigación fue definida como la capacidad de ubicarse en un puesto laboral acorde a su perfil profesional en el menor tiempo posible y en el mayor cargo al mejor sueldo.

\section{METODOLOGÍA}

\section{Tipo y diseño de investigación}

La investigación fue de tipo correlacional, y aplicó un diseño de tipo no experimental de corte transversal.

\section{Unidad de análisis}

La unidad de análisis estuvo representada por cada egresado de la facultad de Administración de la Universidad Nacional Mayor de San Marcos.

\section{Población de estudio}

La población de esta investigación estuvo constituida por todos los egresados de la facultad de Administración de la Universidad Nacional Mayor de San Marcos.

\section{Selección de muestra}

La muestra se determinó teniendo en cuenta el universo de egresados de la facultad de Administración. Para esta investigación, se hizo una estratificación aplicando el criterio de conveniencia. Se 
dividió en dos estratos, el primero conformado por los egresados pertenecientes a la base 2009 de administración de negocios internacionales y otro conformado por los demás egresados. Esta estratificación se debió a una mayor disponibilidad hacia la investigación que tenía el primer estrato.

\section{Tamaño de la muestra}

La población del primer estrato es de alrededor de 96.

$$
\frac{\mathrm{NZ}_{\propto / 2}^{2} \mathrm{P}(1-\mathrm{P})}{(\mathrm{N}-1) \mathrm{e}^{2}+\mathrm{Z}_{\propto / 2}^{2} \mathrm{P}(1-\mathrm{P})}
$$

Dónde:

Con un nivel de significancia de $95 \%$.

$$
\begin{aligned}
& Z=1.96 \\
& N=96 \\
& P=0.97 \\
& 1-P=0.03 \\
& E=0.05
\end{aligned}
$$

Donde $n=30.72483196$ que se considera como 31 .

Y por el criterio de conveniencia, para el segundo estrato se decidió considerar la misma cantidad muestral.

La muestra se determinó teniendo en cuenta el universo de egresados de la facultad de Administración.

\section{Técnica de recolección de datos}

Para el estudio se utilizó como técnica de recolección de datos el cuestionario. El cuestionario aplicativo se formuló ad hoc de acuerdo a los indicadores planteados para llegar así a los objetivos. Se recabo respuestas empleando la encuesta virtual facilitada por la plataforma de Google.

\section{Análisis e interpretación de resultados}

Para el procesamiento de la información recabada a través de la encuesta ad-hoc, se empleó el software estadístico SPSS versión 24. Una vez procesada la información se realizó el análisis de normalidad de datos para determinarla aplicación de los estadísticos pertinentes de esta manera la interpretación se dio mediante cuadros estadísticos, gráficos, ilustraciones, específicamente se ha utilizado la Chi cuadrada, t de student y la prueba de Levene.

\section{RESULTADOS}

A continuación, se presentan gráficamente los resultados:

\section{Sexo}

Se evidencia en la figura 1 , que el $55.4 \%$ de la muestra eran mujeres y la diferencia varones.

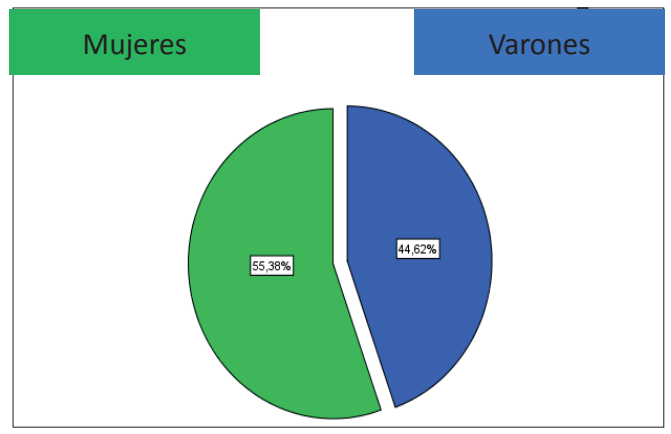

Figura 1. Sexo

Fuente: Elaboración propia

\section{Grados y títulos}

Se puede apreciar en la figura 2, de la muestra $7.7 \%$ se mantuvo como egresado. El $36.9 \%$ tuvo el grado de bachiller, y $55.4 \%$ era licenciado.

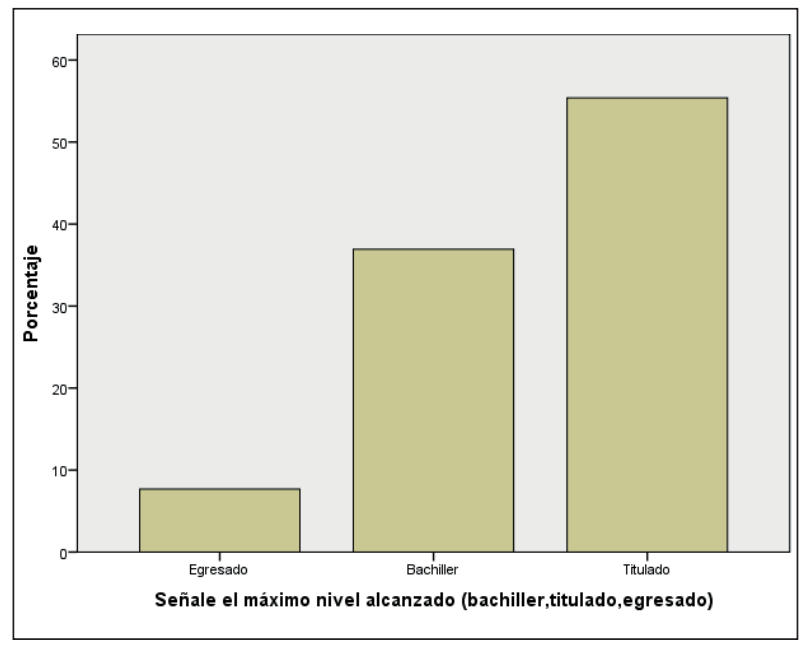

Figura 2. Grados y títulos alcanzados Fuente: Elaboración propia

\section{Edad}

Se muestra en la figura 3 , la edad promedio de la muestra era de 26 años.

\section{Sueldo}

Se observa en la figura 4, estadísticamente el sueldo promedio era de S/. 3 100.00. 


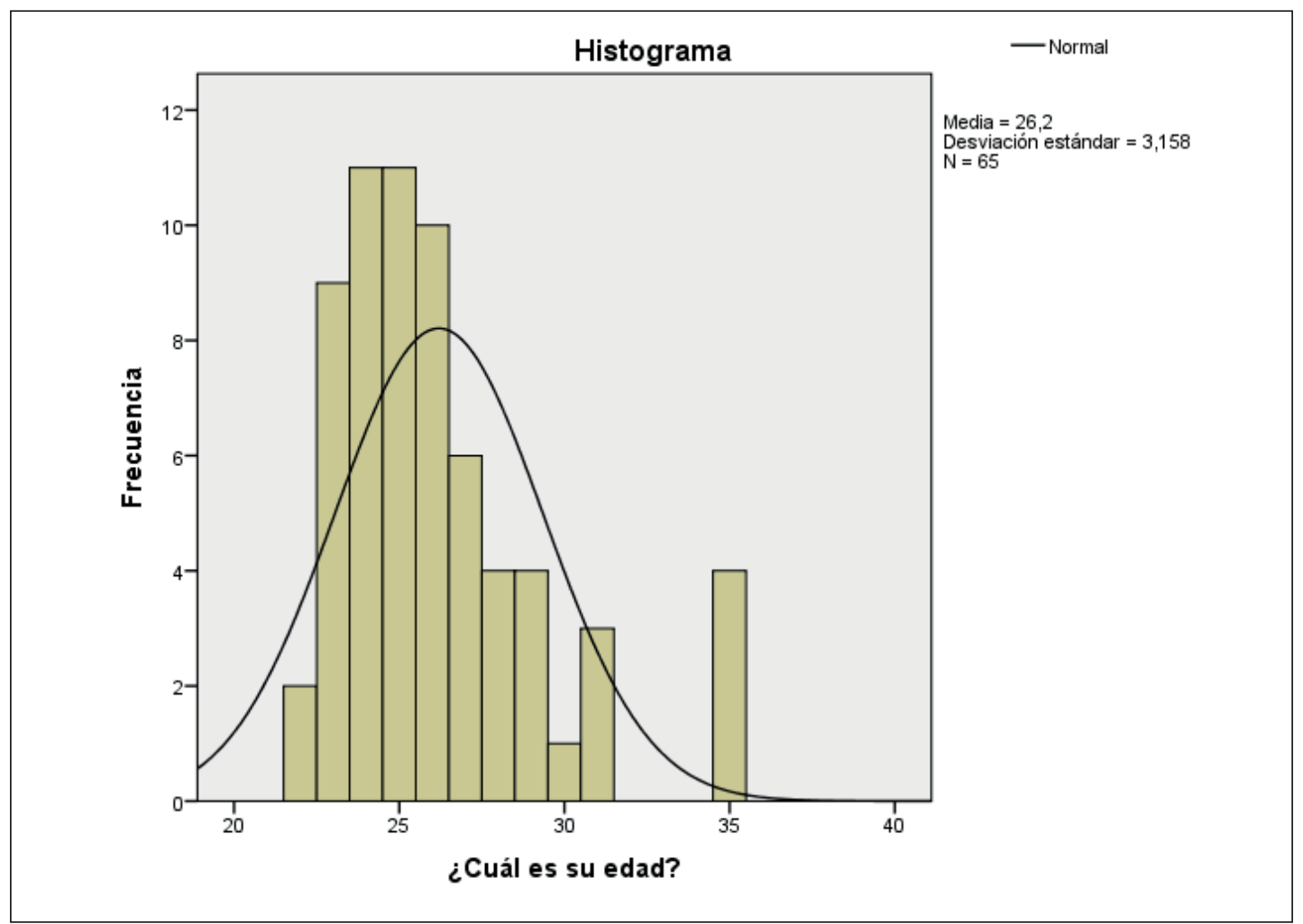

Figura 3. Edad

Fuente: Elaboración propia

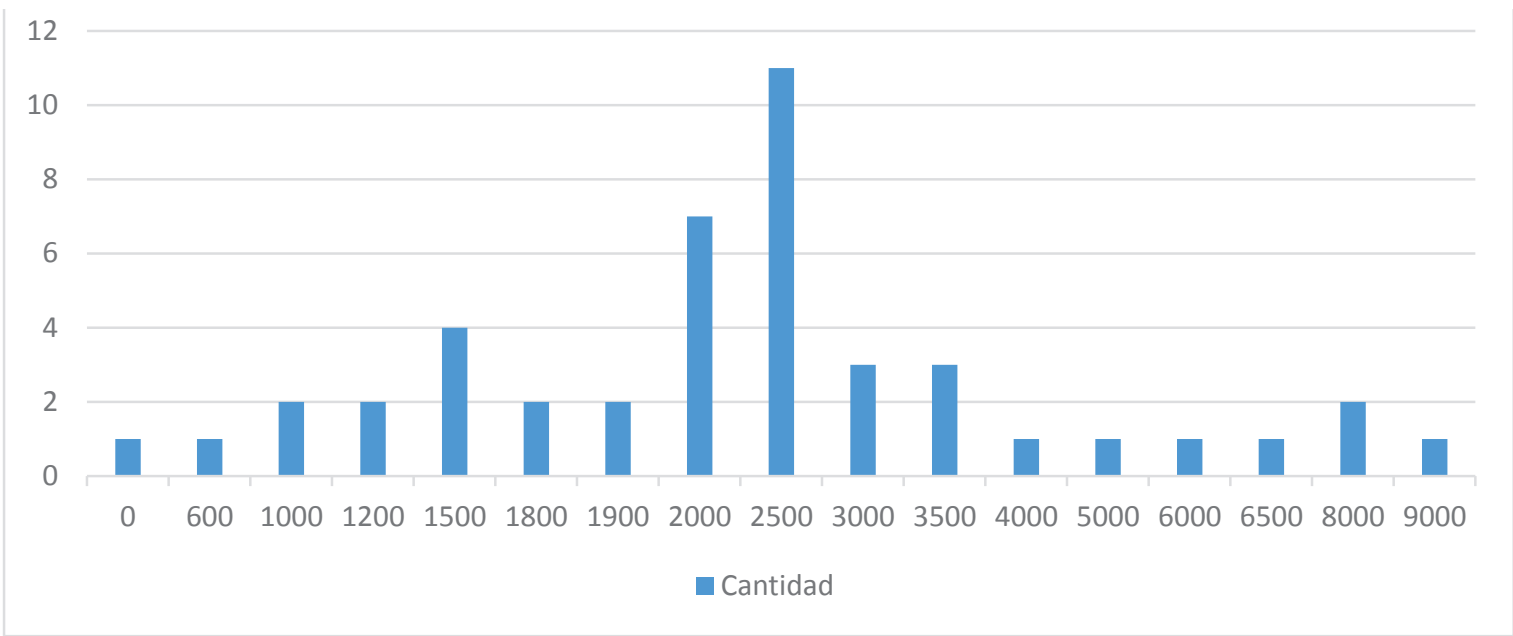

Figura 4. Sueldo

Fuente: Elaboración propia

\section{Beneficios de obtener el Bachiller}

Se muestra en la tabla 1 , al $27.3 \%$ de los que obtuvieron el grado de bachiller consiguieron un trabajo. Al $25.5 \%$ les significó un incremento salarial. Al $9.1 \%$ les significó un ascenso laboral, y al $38.2 \%$ no les reportó ningún efecto.

\section{Beneficios de obtener el Título Profesional}

Se observa en la tabla 2 , al $6.5 \%$ de los que obtuvieron la titulación consiguieron un trabajo. Al $38.7 \%$ les significó un incremento salarial. Al 6.5\% les significó un ascenso laboral, y al $48.4 \%$ no les reportó ningún efecto. 
Tabla 1. Beneficios de obtener el Bachiller

\begin{tabular}{|l|c|c|c|c|}
\hline Beneficios & Frecuencia & Porcentaje & Porcentaje de bachilleres & $\begin{array}{c}\text { Porcentaje total de la } \\
\text { muestra }\end{array}$ \\
\hline Conseguir un trabajo & 15 & 23.1 & 27.3 & 27.3 \\
\hline Un incremento salarial & 14 & 21.5 & 25.5 & 52.7 \\
\hline Un ascenso laboral & 5 & 7.7 & 9.1 & 61.8 \\
\hline No tuvo ningún efecto & 21 & 32.3 & 38.2 & 100.0 \\
\hline Total & 55 & 84.6 & 100.0 & \\
\hline Sólo se mantienen como egresados & 10 & 15.4 & & \\
\hline Total & 65 & 100.0 & & \\
\hline
\end{tabular}

Fuente: Elaboración propia

Tabla 2. Beneficios de obtener el Título Profesional

\begin{tabular}{|l|c|c|c|c|}
\hline Beneficios & Frecuencia & Porcentaje & Porcentaje válido & Porcentaje acumulado \\
\hline Conseguir un trabajo & 2 & 3.1 & 6.5 & 6.5 \\
\hline Un incremento salarial & 12 & 18.5 & 38.7 & 45.2 \\
\hline Un ascenso laboral & 2 & 3.1 & 6.5 & 51.6 \\
\hline No tuvo ningún efecto & 15 & 23.1 & 48.4 & 100.0 \\
\hline Total & 31 & 47.7 & 100.0 & \\
\hline No se titularon & 34 & 52.3 & & \\
\hline Total & 65 & 100.0 & & \\
\hline
\end{tabular}

Fuente: Elaboración propia

\section{Cursos de especialización}

Se puede apreciar en la tabla 3, cerca del $24.07 \%$ no tuvieron un curso de especialización. Aproximadamente el $22.22 \%$ tuvo un curso de especialización. Alrededor de $27.78 \%$ ha llevado 2 cursos de especialización, en torno al $11.11 \%$ tuvieron 3 curso de especialización. Finalmente; más o menos el $14.81 \%$ tuvo 4 cursos de especialización.

Tabla 3. Cantidad de cursos de especialización

\begin{tabular}{|c|c|c|}
\hline & Cursos de Especialización & Porcentaje válido \\
\hline 0 & 13 & 24.1 \\
\hline 1 & 12 & 22.2 \\
\hline 2 & 15 & 27.8 \\
\hline 3 & 6 & 11.1 \\
\hline 4 & 8 & 14.8 \\
\hline Total & 54 & 100.0 \\
\hline
\end{tabular}

Fuente: Elaboración propia

\section{Cantidad de seminarios o congresos}

Se puede observar en la tabla 4 , cerca del $28.57 \%$ no ha llevado seminarios o congresos. Aproximadamente el $33.93 \%$ tiene un seminario o congreso.
Alrededor de $21.43 \%$ ha llevado 2 seminarios o congresos, en torno al $1.79 \%$ tuvo 3 seminarios o congresos. Finalmente; más o menos el $14.29 \%$ tuvo 4 seminarios o congresos.

Tabla 4. Cantidad de seminarios o congresos

\begin{tabular}{|c|c|c|}
\hline & Seminarios o congresos & Porcentaje válido \\
\hline 0 & 16 & 28.6 \\
\hline 1 & 19 & 33.9 \\
\hline 2 & 12 & 21.4 \\
\hline 3 & 1 & 1.8 \\
\hline 4 & 8 & 14.3 \\
\hline Total & 56 & 100.0 \\
\hline
\end{tabular}

Fuente: Elaboración propia

\section{Cantidad de cursos de informática}

Se puede apreciar en la tabla 5, cerca del $5.08 \%$ no ha llevado algún curso de informática. Aproximadamente el $33.90 \%$ tuvo un curso de informática. Alrededor del $32.20 \%$ ha llevado 2 cursos de informática, en torno al $13.56 \%$ tiene 3 cursos de informática. Finalmente; más o menos el $15.25 \%$ tuvo 4 cursos de informática. 
Tabla 5. Cantidad de cursos de informática

\begin{tabular}{|c|c|c|}
\hline & Cursos de informática & Porcentaje válido \\
\hline 0 & 3 & 5.1 \\
\hline 1 & 20 & 33.9 \\
\hline 2 & 19 & 32.2 \\
\hline 3 & 8 & 13.6 \\
\hline 4 & 9 & 15.3 \\
\hline Total & 59 & 100.0 \\
\hline
\end{tabular}

Fuente: Elaboración propia

\section{Cantidad de diplomados}

Se observa en la tabla 6 , cerca del $45.45 \%$ no ha llevado algún diplomado. Aproximadamente el $27.27 \%$ tiene un diplomado. Alrededor de $18.18 \%$ ha llevado 2 diplomados, en torno al 3.64\% tuvo 3 diplomados. Finalmente; más o menos el $5.45 \%$ tuvo 4 diplomados.
Tabla 6. Cantidad de Diplomados

\begin{tabular}{|c|c|c|}
\hline & Diplomados & Porcentaje válido \\
\hline 0 & 25 & 45.5 \\
\hline 1 & 15 & 27.3 \\
\hline 2 & 10 & 18.2 \\
\hline 3 & 2 & 3.6 \\
\hline 4 & 3 & 5.5 \\
\hline Total & 55 & 100.0 \\
\hline
\end{tabular}

Fuente: Elaboración propia

\section{Cruce de variables}

La tabla 7 muestra el cruce de los indicadores del capital humano con los indicadores de la variable empleabilidad y señala si existe relación entre ellos.

Tabla 7. Cruce de variables

\begin{tabular}{|c|c|c|c|c|c|c|}
\hline Variables & Relación & Indicador & Salario & $\begin{array}{c}\text { Tiempo en conseguir } \\
\text { empleo }\end{array}$ & Aumento Salarial & Ascenso Laboral \\
\hline \multirow{7}{*}{$\begin{array}{l}\text { Nombre: } \\
\text { Capital } \\
\text { humano y } \\
\text { Empleabi- } \\
\text { lidad }\end{array}$} & \multirow{5}{*}{$\begin{array}{l}\text { Grado } \\
\text { Académico } \\
\text { y tipos de } \\
\text { preparación } \\
\text { académica y } \\
\text { su relación } \\
\text { con la } \\
\text { empleabiliad } \\
\text { del egresado }\end{array}$} & $\begin{array}{l}\text { Diploma } \\
\text { Académico }\end{array}$ & $\begin{array}{l}\text { Existe una diferencia sig- } \\
\text { nificativa entre la media } \\
\text { del salario del grupo de } \\
\text { bachilleres y la media } \\
\text { del salario del grupo de } \\
\text { titulados. }\end{array}$ & $n / a$ & $\mathrm{n} / \mathrm{a}$ & $\mathrm{n} / \mathrm{a}$ \\
\hline & & $\begin{array}{l}\text { Cursos de } \\
\text { Informática }\end{array}$ & $\begin{array}{l}\text { No existe correlación } \\
\text { entre el número de capa- } \\
\text { citaciones en cursos de } \\
\text { informática y el salario } \\
\text { bruto percibido. }\end{array}$ & $\begin{array}{l}\text { No existe correlación entre } \\
\text { el número de capacitacio- } \\
\text { nes en cursos de informá- } \\
\text { tica y el tiempo promedio } \\
\text { en conseguir empleo }\end{array}$ & \begin{tabular}{|l|} 
No existe correlación \\
entre el número de \\
aumentos salariales \\
y el número de cur- \\
sos de informática. \\
\end{tabular} & $\begin{array}{l}\text { No existe correlación } \\
\text { entre el número de } \\
\text { capacitaciones en } \\
\text { cursos de informática } \\
\text { y número de ascensos } \\
\text { laborales recibidos } \\
\end{array}$ \\
\hline & & Diplomados & $\begin{array}{l}\text { No existe correlación } \\
\text { entre el número de } \\
\text { capacitaciones en diplo- } \\
\text { mados y el salario bruto } \\
\text { percibido }\end{array}$ & $\begin{array}{l}\text { Existe correlación entre el } \\
\text { número de capacitaciones } \\
\text { en diplomados y el tiempo } \\
\text { en promedio que se tarda } \\
\text { en conseguir un empleo }\end{array}$ & \begin{tabular}{|l|} 
No existe correlación \\
entre el número \\
de capacitaciones \\
en diplomados y el \\
aumento salarial \\
recibido
\end{tabular} & $\begin{array}{l}\text { Existe correlación entre } \\
\text { el número de capacita- } \\
\text { ciones en diplomados y } \\
\text { el número de ascensos } \\
\text { laborales recibidos }\end{array}$ \\
\hline & & $\begin{array}{l}\text { Seminarios } \\
\text { o Congresos }\end{array}$ & $\begin{array}{l}\text { Existe correlación entre } \\
\text { el número de capacita- } \\
\text { ciones en seminarios o } \\
\text { congresos y el salario } \\
\text { bruto percibido. }\end{array}$ & $\begin{array}{l}\text { Existe correlación entre el } \\
\text { número de capacitaciones } \\
\text { en seminarios o congresos } \\
\text { y el tiempo promedio en } \\
\text { conseguir un empleo. }\end{array}$ & $\begin{array}{l}\text { Existe correlación } \\
\text { entre el número de } \\
\text { capacitaciones en } \\
\text { seminarios o congre- } \\
\text { sos y el salario bruto } \\
\text { percibido. }\end{array}$ & $\begin{array}{l}\text { No existe correlación } \\
\text { entre el número de } \\
\text { capacitaciones en } \\
\text { seminarios o congresos } \\
\text { y el número de veces } \\
\text { que se ha recibido un } \\
\text { ascenso laboral. } \\
\end{array}$ \\
\hline & & $\begin{array}{l}\text { Cursos de Es- } \\
\text { pecialización }\end{array}$ & $\begin{array}{l}\text { Existe correlación entre } \\
\text { el número de especiali- } \\
\text { zación y el salario bruto } \\
\text { percibido. }\end{array}$ & $\begin{array}{l}\text { No existe correlación entre } \\
\text { el número de capacitacio- } \\
\text { nes en especializaciones } \\
\text { y el tiempo que tarda en } \\
\text { conseguir un empleo. }\end{array}$ & \begin{tabular}{|l|} 
Existe correlación \\
entre el número de \\
capacitaciones en \\
especializaciones y \\
el número de aumen- \\
to salarial \\
\end{tabular} & $\begin{array}{l}\text { Existe correlación entre } \\
\text { el número de capaci- } \\
\text { taciones en especiali- } \\
\text { zaciones y el número } \\
\text { de ascensos laborales } \\
\text { recibidos }\end{array}$ \\
\hline & $\begin{array}{l}\text { Experiencia } \\
\text { laboral y su } \\
\text { relación con } \\
\text { la emplea- } \\
\text { bilidad del } \\
\text { egresado }\end{array}$ & $\begin{array}{l}\text { Experiencia } \\
\text { laboral }\end{array}$ & $\begin{array}{l}\text { Existe correlación entre } \\
\text { el tiempo de experiencia } \\
\text { laboral relacionada a } \\
\text { la profesión y el salario } \\
\text { bruto percibido. }\end{array}$ & $\mathrm{n} / \mathrm{a}$ & \begin{tabular}{|l|} 
No existe correlación \\
entre el tiempo de \\
experiencia laboral \\
relacionada a la \\
profesión y el número \\
de veces de aumento \\
promedio salarial
\end{tabular} & $\begin{array}{l}\text { No existe correlación } \\
\text { entre el tiempo de } \\
\text { experiencia laboral rela- } \\
\text { cionada a la profesión y } \\
\text { el número de ascensos } \\
\text { laborales recibidos }\end{array}$ \\
\hline & & Carrera laboral & $\begin{array}{l}\text { Existe correlación entre } \\
\text { el número de trabajos } \\
\text { relacionadas a la pro- } \\
\text { fesión y el salario bruto } \\
\text { percibido. }\end{array}$ & $\begin{array}{l}\text { No existe correlación entre } \\
\text { el número de trabajos } \\
\text { relacionada a la profesión } \\
\text { y el tiempo promedio en el } \\
\text { que se ha conseguido un } \\
\text { empleo }\end{array}$ & \begin{tabular}{|l|} 
No existe correlación \\
entre el número de \\
trabajos relacionadas \\
a la profesión y el \\
número de veces de \\
aumento promedio \\
salarial
\end{tabular} & $\begin{array}{l}\text { No existe correlación } \\
\text { entre el número de } \\
\text { trabajos relacionadas a } \\
\text { la profesión y el número } \\
\text { de ascensos laborales } \\
\text { recibidos }\end{array}$ \\
\hline
\end{tabular}




\begin{tabular}{|c|c|c|c|c|c|c|}
\hline Variables & Relación & Indicador & Salario & $\begin{array}{c}\text { Tiempo en conseguir } \\
\text { empleo }\end{array}$ & Aumento Salarial & Ascenso Laboral \\
\hline & \multirow[t]{2}{*}{$\begin{array}{l}\text { Variables } \\
\text { socio demo- } \\
\text { gráficas y su } \\
\text { relación con } \\
\text { la emplea- } \\
\text { bilidad del } \\
\text { egresado }\end{array}$} & Sexo & $\begin{array}{l}\text { No existe una diferencia } \\
\text { significativa entre el sala- } \\
\text { rio bruto medio del sexo } \\
\text { masculino y el salario } \\
\text { bruto medio del sexo } \\
\text { femenino. }\end{array}$ & $\begin{array}{l}\text { Existe una diferencia sig- } \\
\text { nificativa entre el tiempo } \\
\text { promedio en conseguir } \\
\text { empleo por el sexo mas- } \\
\text { culino y el tiempo prome- } \\
\text { dio en conseguir empleo } \\
\text { por el sexo femenino. }\end{array}$ & $\begin{array}{l}\text { Existe una dife- } \\
\text { rencia significativa } \\
\text { entre el número de } \\
\text { veces promedio de } \\
\text { aumento salarial por } \\
\text { el sexo masculino y } \\
\text { el número de veces } \\
\text { promedio de aumen- } \\
\text { to salarial por el sexo } \\
\text { femenino }\end{array}$ & $\begin{array}{l}\text { Existe una diferencia } \\
\text { significativa entre la } \\
\text { media del número de } \\
\text { ascensos laborales } \\
\text { recibidos por del sexo } \\
\text { masculino y la media } \\
\text { del número de ascen- } \\
\text { sos laborales recibidos } \\
\text { del sexo femenino. }\end{array}$ \\
\hline & & Edad & $\begin{array}{l}\text { Existe correlación entre } \\
\text { la edad y el salario bruto } \\
\text { percibido }\end{array}$ & $\begin{array}{l}\text { No existe correlación entre } \\
\text { la edad y el tiempo prome- } \\
\text { dio que tardó en conseguir } \\
\text { un empleo }\end{array}$ & $\begin{array}{l}\text { No existe correlación } \\
\text { entre la edad y el nú- } \\
\text { mero de veces que } \\
\text { recibió un aumento } \\
\text { salarial }\end{array}$ & $\begin{array}{l}\text { Existe correlación entre } \\
\text { la edad y número de } \\
\text { veces que ha recibido } \\
\text { un ascenso laboral }\end{array}$ \\
\hline
\end{tabular}

Fuente: Elaboración propia

\section{DISCUSIÓN}

Según los resultados antes mostrados se lleva a cabo la contrastración de los mismos, a saber:

El rango académico obtenido al egresar no ha influido sobre el salario, no obstante, el título profesional sí influye en el salario. Esto guarda relación con los hallazgos realizados con el trabajo de investigación Ríos (2015) señala que solo el $2.2 \%$ de los egresados en Administración lo precisan como requisito principal de acceso laboral después del primer trabajo, mientras que el $0.0 \%$ lo requiere como requisito principal de acceso siendo su primer trabajo al que postulen. Con respecto al diplomado, éste no ha influido para obtener un mayor salario, sin embargo, si influye para conseguir ascensos en la carrera profesional.

Por otra parte, los seminarios, congresos o cursos de especialización tienen un impacto directamente proporcional sobre el salario y los cursos de especialización tienen una relación positiva sobre el número de ascensos laborales. Es relevante mencionar que los cursos de informática son herramientas buscadas por los egresados pero estas no influyen estadísticamente en la empleabilidad, como también lo concluye el mencionado trabajo de investigación, esto se ve respaldado por los resultados obtenidos de Ríos (2015) señala que las habilidades y competencias técnicas adquiridas son requisito principal de acceso laboral para el primer trabajo con $5.9 \%$ y para después del primer trabajo con $8.1 \%$ siendo las valoraciones más altas dentro de los requisitos para inserción laboral en dicho estudio.

Se infiere que a mayor experiencia laboral, existe un mayor salario, lo mismo que si se ha desempe- ñado en trabajos relacionados con lo estudiado. Aunque estos factores no repercuten sobre el número de ascensos laborales, estas premisas encuentran relacionadas con los hallazgos encontrados en la investigación de Ríos (2015) que muestra que el grueso de los egresados en la carrera profesional de Administración el $6.1 \%$ perciben entre $\mathrm{S} / .2001-3000$, un $2.6 \%$ entre $\mathrm{S} / .3001-5000$ y un $0.9 \%$ entre $\mathrm{S} / .5001$ a más de ingresos económicos mensuales. Asimismo, Tito, P., Pereda, F., Vilcabana, P. (2008) señalan que a mayor edad y años de egresado son determinantes para llevar a cabo funciones importantes en la especialidad. Por otra parte, el tiempo en que un profesional se demora en conseguir un empleo no se ve estadísticamente influenciado por la variable capital humana, esto es, formación y experiencia laboral. Esto, condice con los hallazgos de Ríos (2015) que señala que la demora en acceso al trabajo para un egresado de la carrera profesional de Administración es de tres meses, sea su primer trabajo o después del primer trabajo.

Por otro lado, se tiene como hallazgo de esta investigación que aquellos que tienen un mayor número de diplomados, seminarios o congresos se demoran más en conseguir un trabajo. Esto podría deberse a que el periodo que transcurre entre trabajo y trabajo puede ser empleado para capacitarse. Esto, se explicaría porque los egresados manifestaron que el tiempo para encontrar un nuevo trabajo es mayor. Aunque no existe una diferencia estadísticamente significativa entre el salario recibido por los varones y por las mujeres. Por un lado, los varones reciben más aumentos salariales y tienen un mayor número de ascensos laborales mientras que las mujeres se demoran menos tiempo en conseguir un trabajo. Estas inferencias pueden ser respaldadas por los resultados y conclusiones propias de la investiga- 
ción de Ríos. (2015) quien señala que en relación al género, el posicionamiento y expectativas los egresados de la carrera profesionales analizadas muestran una incorporación diferenciada en el mercado laboral.

De la investigación realizada estadísticamente se ha comprobado que existe una relación entre mayor edad y un salario más alto y entre mayor edad y un mayor número de ascensos. Sin embargo, la edad no repercute en el tiempo en conseguir un trabajo, ni positiva ni negativamente.

Finalmente, comparado con los antecedentes, se corrobora que la mayoría de los egresados están laborando y con el trascurrir del tiempo, a medida que van capacitándose y acumulando experiencia obtienen mayores y diversos beneficios laborales.

\section{CONCLUSIONES}

Los resultados de la investigación a los egresados de la Universidad Nacional Mayor de San Marcos nos conllevan a señalar que:

Los indicadores de la variable independiente: capital humano, tuvo diversos efectos sobre los indicadores de la empleabilidad.

- La formación académica influye sobre el salario, el ascenso laboral, el tiempo en conseguir un empleo y la obtención de un aumento salarial.

- El tiempo de experiencia laboral especifica acumulada influye sobre los ingresos más no en otros indicadores de la variable empleabilidad.

\section{RECOMENDACIONES}

Se recomienda obtener el título profesional a los meses de culminada la carrera esto contribuirá a la empleabilidad según lo demostrado, ya sea vía tesis o examen de suficiencia, lo cual estará sujeto a la normativa universitaria vigente.

Asimismo, se recomienda tomar diplomados, seminarios o cursos cortos de forma voluntaria que demostrarán el interés por parte del egresado de ascender laboralmente ante su empleador.

En tanto las capacitaciones informáticas la Universidad debe brindar cursos de software empresarial y enseñar lenguajes de programación para la creación de aplicativos orientados a los negocios, así como efectuar convenios estratégicos para la certificación internacional del idioma y especialidades en las ramas de la carrera profesional. En específico, la implementación de diplomados sobre Gestión de proyectos y Contrataciones con el Estado, por ser los diplomados más requeridos por diversas entidades. Esto permitirá futuros ascensos laborales y consiguientemente ascensos salariales como lo ha demostrado la presente investigación.

Se recomienda también que el egresado inicie a laborar por lo menos un año antes de su egreso de manera que contará con experiencia base en el rubro que haya elegido. Es importante señalar que el egresado debe decidir con cierta anticipación no menor a 03 meses, al momento de buscar una oportunidad laboral o reinsertarse laboralmente, porque se ve sujeto a la competencia permanente.

La universidad debe establecer nuevos canales de comunicación con los egresados que permitan de forma permanente y eficaz conocer sobre su situación laboral.

\section{AGRADECIMIENTO}

Este trabajo de investigación fue posible gracias a la participación de los egresados de la Facultad de Ciencias Administrativas de la Universidad Nacional Mayor de San Marcos.

\section{REFERENCIAS BIBLIOGRÁFICAS}

Améstica, L., Llinas, X., y Sánchez, I. (2014). Return of Higher Education in Chile: Effect on Social Mobility through Differences-inDifferences estimator. Formación universitaria, 7(3), 23-32. https://dx.doi.org/10.4067/S071850062014000300004

Ananias, R. (2014). Sabiendo lo que hay que saber en Ciencias de la Madera revalorizado en la sociedad del conocimiento. Maderas. Ciencia y tecnología, 16(3), 273-274. https://dx.doi. org/10.4067/S0718-221X2014000300001

Becker, G. (2002) The age of human capital. Education in the Twenty-first Century.

Geeregat, O., Cifuentes G., y Villarroel F, M. (2016). Factores que inciden en las condiciones de empleabilidad de los egresados de pedagogía. Actualidades Investigativas en Educación, 16 (1), 1-19. http://dx.doi.org/10.15517/aie. v16i1.21935 
Conferencia de Ministros europeos responsables de educación superior. (2009). Comunicado de la Conferencia de Ministros europeos responsables de educación superior. Recuperado de http://media.ehea.info/ file/2009_Leuven_Louvain-la-Neuve/06/1/ Leuven_Louvain-la-Neuve_Communique April_2009_595061.pdf

López, V, Nevado, D., y Baños, J. (2008). Indicador sintético de capital intelectual: humano y estructural. Un tactor de competitividad. EURE (Santiago), 34(101), 45-70. https://dx.doi. org/10.4067/S0250-71612008000100003

Mallado, C, Parejo, S, y Bozal, R. (2016). Competencias implicadas en la empleabilidad: una propuesta desde la igualdad de género. Faces de Eva. Estudos sobre a Mulher, (35), 55-71. Recuperado de http://www.scielo.mec. pt/scielo.php?script=sci_arttext\&pid=S0874$68852016000100006 \& \operatorname{lng}=$ pt\&tIng=en

Martínez J. (2011) La empleabilidad: una competencia personal y una responsabilidad social. Contribuciones a las Ciencias Sociales (14) Noviembre.

Martínez, J. (2012) Turismo, la competencia de empleabilidad de los futuros profesionales del turismo. TURYDES 5(12) 1-27

Paravic, T. (2010). Enfermería y globalización. Ciencia y enfermería, 16(1), 9-15. https://dx.doi. org/10.4067/S0717-95532010000100002
Ramírez, D. (2007) Capital humano como factor de crecimiento económico: caso departamento de caldas (Colombia). 1983 2003. Universidad de Manizales.

Ríos, J. (2015) Inserción de los profesionales sanmarquinos en el Mercado laboral global, 2010-2015: Un studio de casos hacia una vision sociohistórica prospectiva. Investigaciones sociales. Vol.20 №37, pp.149-164, [2016, UNMSM-IIHS, LIMA, PERÚ.

Riveros, L, y Báez, G. (2014). Chile y la OCDE: La dicotomía entre lo macroeconómico y el desarrollo humano. Estudios internacionales (Santiago), 46(179), 9-34. https://dx.doi. org/10.5354/0719-3769.2014.35461

Rodríguez, E, y Palma, Á. (2010). Desafíos de la educación superior en la economía del conocimiento. Ingeniare. Revista chilena de ingeniería, 18(1), 8-14. https://dx.doi. org/10.4067/S0718-33052010000100002

Romero, E. y Chavarría, L. (2009) Inserción en el mercado laboral del egresado sanmarquino. C.Lima - Perú. Editorial Universidad Nacional Mayor de San Marcos.

Schultz, T. (1975) Human Capital: Policy Issues And Research Opportunities. Economic Research: Retrospect and prospect. 6, 1-84

Tito, P., Pereda, F., Vilcabana, P. (2008). Empleabilidad de egresados de la Facultad de Ciencias Administrativas de la UNMSM. Gestión en el Tercer Milenio, 11(22), 59. 